\title{
Correction to: UK Business and Financial Cycles Since 1660
}

\section{Correction to:}

N. Dimsdale and R. Thomas,

UK Business and Financial Cycles Since 1660, https://doi.org/10.1007/978-3-030-26346-1

The original version of the book unfortunately had some chapter-wise corrections post publication. The corrections have been incorporated in the book.

The updated versions of the chapters can be found at https://doi.org/10.1007/978-3-030-26346-1_2 https://doi.org/10.1007/978-3-030-26346-1_3 https://doi.org/10.1007/978-3-030-26346-1_4 https://doi.org/10.1007/978-3-030-26346-1_6 https://doi.org/10.1007/978-3-030-26346-1 